\title{
Goodpasture's syndrome in a patient with two endocrine tumours
}

\author{
R.F.T. McMahon', W. Lawler' ${ }^{1}$, D.J. O’Donoghue ${ }^{2}$ and F.W. Ballardie' \\ ${ }^{1}$ Department of Pathology, University of Manchester, Manchester M13 9PT, and ${ }^{2}$ Department of Renal Medicine, \\ Manchester Royal Infirmary, Manchester, UK.
}

\begin{abstract}
Summary: We describe the occurrence of two anatomically separate and histologically distinct endocrine tumours of bronchus and pancreas in a 64 year old woman with biopsy and serology proven Goodpasture's syndrome. The association between anti-glomerular basement membrane antibody disease and multiple endocrine tumours has not been reported previously; possible pathogenetic mechanisms are discussed.
\end{abstract}

\section{Introduction}

We present a woman with Goodpasture's syndrome who died from myocardial infarction. At autopsy, two anatomically separate and histologically distinct endocrine tumours were observed - a bronchial carcinoid and a pancreatic islet cell tumour. The association between Goodpasture's syndrome and multiple endocrine tumours has not been reported before, and although this association may be purely coincidental, it is possible that tumour-related antigens secreted by one or both neoplasms may cross-react with alveolar/ glomerular capillary basement membranes, thus causing the classical anti-glomerular basement membrane antibody disease.

\section{Case report}

A 64 year old housewife with long-standing winter bronchitis presented with a 3-month history of persistent cough, increasing dyspnoea, anorexia without other gastrointestinal symptoms and $12 \mathrm{~kg}$ weight loss. Her cough produced purulent, occasionally blood-stained sputum but no major haemoptysis. She was a non-smoker and there was no exposure to noxious fumes. Physical examination revealed a thin white woman who was febrile $\left(38.2^{\circ} \mathrm{C}\right)$, pale, dyspnoeic at rest and tachypnoeic; the heart rate was 100 per minute, blood pressure $150 / 80 \mathrm{mmHg}$ and there was mild bilateral ankle oedema. Chest auscultation revealed coarse bilateral basal inspiratory crackles and scattered expiratory rhonchi. Urine contained ++++ protein, numerous erythrocytes and many

\footnotetext{
Correspondence: R.F.T. McMahon, B.Sc., M.B., M.R.C.Path.

Accepted: 9 February 1989
}

red cell and granular casts. Haemoglobin was $7.4 \mathrm{~g} / \mathrm{dl}$, white cell count $26.7 \times 10^{9} / 1$ and erythrocyte sedimentation rate $>140 \mathrm{~mm} / \mathrm{h}$; sodium was $131 \mathrm{mmol} / 1$, potassium $3.2 \mathrm{mmol} / 1$, urea $41.6 \mathrm{mmol} / \mathrm{l}$, creatinine $653 \mu \mathrm{mol} / 1$, calcium $2.04 \mathrm{mmol} / \mathrm{l}$, phosphate $2.99 \mathrm{mmol} / 1$, albumin $27 \mathrm{~g} / \mathrm{l}$, globulins $35 \mathrm{~g} / \mathrm{l}$, glucose $6.3 \mathrm{mmol} / 1$ and urate $0.65 \mathrm{mmol} / 1$. A 24-hour urine of $1270 \mathrm{ml}$ contained $11.4 \mathrm{~g}$ protein; the calculated creatinine clearance was $6 \mathrm{ml} / \mathrm{min}$. Serum immunoglobulins and complement levels were normal. Antiglomerular basement membrane antibodies (anti-GBM abs) were positive at $44 \%$ (normal $<12 \%$ ). Blood cultures were negative; urine culture yielded scanty Escherichia coli and sputum grew Haemophilus parainfluenzae, both sensitive to erythromycin. Chest radiographs showed bilateral mid and lower zone alveolar interstitial infiltrates, more marked on the right side. Ultrasound examination revealed normal sized kidneys with no obstruction. Renal biopsy is discussed below. She was commenced on intravenous erythromycin, daily 4 litre plasmapheresis, cyclophosphamide $(2.5 \mathrm{mg} / \mathrm{kg} / \mathrm{day})$ and prednisolone (60 mg/day). Anti-GBM abs dropped to $34 \%$ and $21 \%$ on consecutive days. Despite prophylactic fresh frozen plasma, haemorrhage from the renal biopsy site occurred after the 3rd exchange; plasmapheresis was then discontinued and 3 units of blood were given. Her renal function improved plasma creatinine fell to $430 \mu \mathrm{mol} / 1$ and daily urine output exceeded 2 litres although anti-GBM abs again increased to $46 \%$; respiratory symptoms improved, but chest radiographs remained unchanged. On the 10th hospital day she complained of severe central chest pain; the electrocardiogram showed a fresh anterior infarct and she suffered a cardiorespiratory arrest. Resuscitation restored cardiac output, but she 
required mechanical ventilation and died 4 days later without regaining consciousness.

\section{Materials and methods}

The renal biopsy was treated routinely: after small fragments had been cut from each end of the core and fixed for electron microscopy, the remainder was bisected, with half prepared for light microscopy ${ }^{1}$ and half frozen, cut at $5 \mu$ and stained with fluoresceinisothiocyanate labelled antibodies against IgG, IgA, $\operatorname{IgM}, \mathrm{C}_{3}$ and $\mathrm{C}_{1 \mathrm{q}}$. The autopsy was performed within 3 hours of death; tissues were fixed in neutral buffered formalin, processed and embedded in paraffin, and sections were cut at $4 \mu$ and stained with haematoxylin and eosin (H\&E). Sections of bronchus and pancreas were stained for reticulin, by the diazonium dye (argentaffin), Masson Fontana (argentaffin) and Grimelius' (argyrophil) methods and for neuronespecific enolase (NSE) and S-100 protein; pancreatic sections were also stained for insulin, glucagon and somatostatin. Later, kidney and bronchial tumour sections were stained for IgG, IgA and IgM (DAKO); insufficient pancreatic islet cell tumour tissue was available for immunoglobulin staining.

\section{Renal biopsy}

The tissue submitted for light microscopy contained 21 glomeruli; four were totally sclerosed; all the remainder showed tuft distortion and compression by cellular capsular crescents, many incorporating fibrin

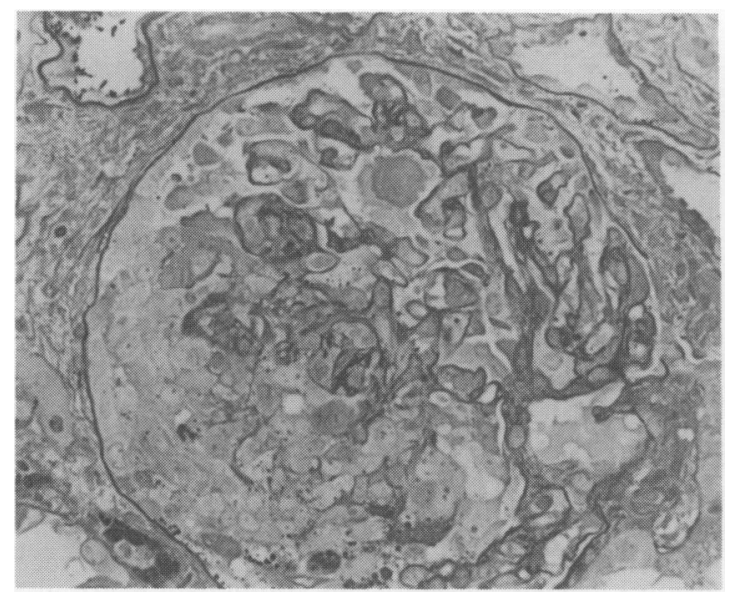

Figure 1 A glomerulus showing a cellular capsular crescent with distortion of the tuft and focal necrosis (haematoxylin and phloxine $\times 264$ ).
(Figure 1); a few tufts also showed small foci of segmental fibrinoid necrosis. Immunofluorescence revealed diffuse linear IgG deposits along basement membranes (Figure 2) with less intense $\mathrm{C}_{3}$ deposition and occasional IgM granules. The appearances were those of a necrotizing crescentic glomerulonephritis due to antiglomerular basement membrane antibody disease.

\section{Autopsy findings}

The heart (390 grams) showed biventricular dilatation and recent anterior papillary muscle infarction.

The lungs showed lower lobe bronchiectasis, early bronchopneumonia and intra-alveolar haemorrhages up to $0.5 \mathrm{~cm}$ in diameter. Although no bronchial tumour was seen macroscopically, histology revealed submucosal clusters and packets of darkly staining, hyperchromatic cells showing only minimal pleomorphism, some of which were in lymphatic channels (Figure 3); argentaffin and argyrophil reactions and stains for NSE, S-100 protein, IgG, IgA and IgM were negative.

In the kidneys (right 200 grams; left 160 grams) many glomeruli were totally sclerosed; most of the remainder were diffusely hypercellular, and crescents were seen in approximately $50 \%$. Staining for $\mathrm{IgG}$ was linear but only weakly positive along peripheral capillary loops.

The pancreas appeared normal macroscopically; histologically, a single tumour focus was seen which comprised uniform cells, with a high nuclearcytoplasmic ratio, somewhat vesicular nuclei and

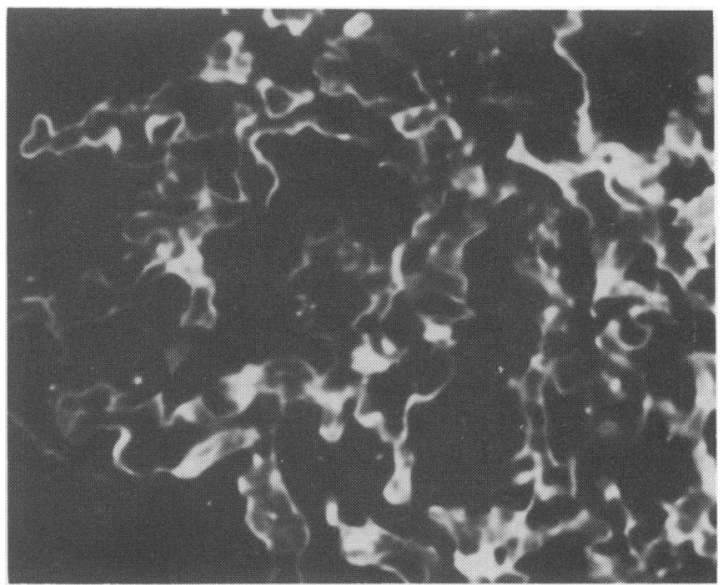

Figure 2 Diffuse linear deposition of IgG along glomerular capillary basement membranes (immunofluorescence $\times 391$ ). 


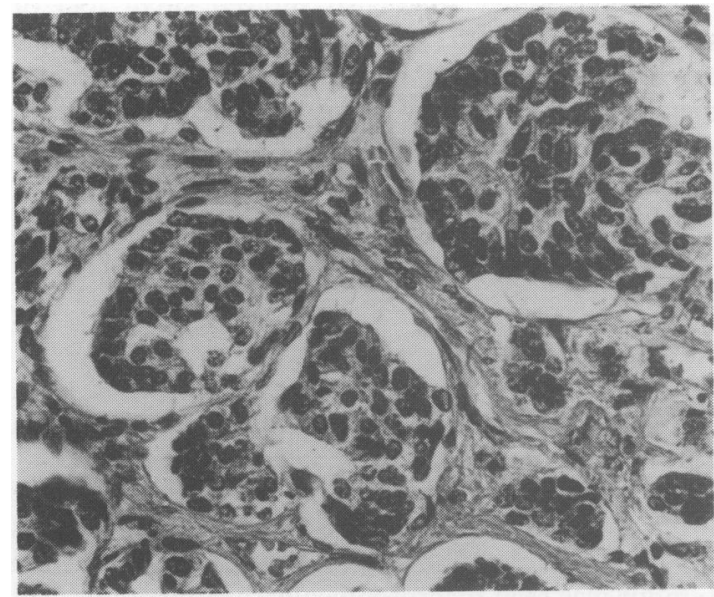

Figure 3 Packets of hyperchromatic cells in the bronchial submucosa, including some within lymphatic channels. (H\& E $\times 264)$.

granular cytoplasm, arranged in a trabecular pattern (Figure 4). Grimelius' stain was positive but argentaffin reactions and stains for NSE, S-100 protein, insulin, glucagon and somatostatin were negative.

\section{Discussion}

Goodpasture's syndrome is rare. It represents 3 out of 25,000 autopsies $(0.012 \%), 0.15 \%$ of all renal biopsies and $0.3 \%$ of all glomerulonephritides in a German series; ${ }^{2}$ in Manchester, it accounts for $0.75 \%$ of all successful non-transplant renal biopsies from an adult population. It is the classical form of anti-glomerular basement membrane antibody disease where linear deposition of IgG and $C_{3}$ is seen along capillary basement membranes, and it is often preceded by haemoptysis or lung haemorrhage due to cross reactivity with alveolar basement membranes. ${ }^{2,3}$ Renal disease presents with microscopic haematuria, slight proteinuria, oliguria and acute renal failure. Untreated, prognosis is poor; treatment with steroids, immunosuppressives and plasmapheresis yields variable results. Transplantation is usually successful when circulating anti-GBM abs are no longer present.

Affected patients are usually young (15-35 years), and there is a male preponderance $(2: 1)$, but it may occur at any age in either sex. The pathogenetic mechanism is antibody attachment to alveolar and glomerular capillary basement membranes, but the reason for initiation of antibody production is unknown. Most cases appear to occur spontaneously,

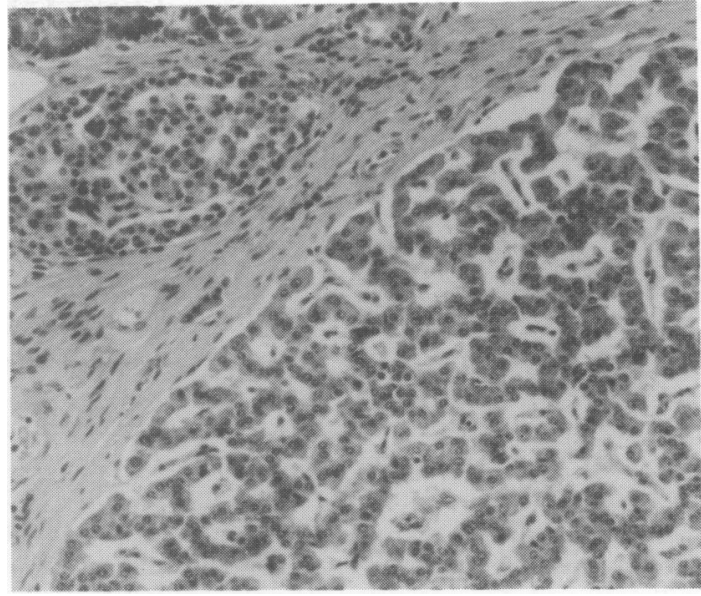

Figure 4 Pancreatic islet cell tumour of trabecular pattern (centre right) with adjacent normal islet (top left). $(H \& E \times 81)$.

but proposed precipitating causes include crossreaction against streptococcal membrane ${ }^{4}$ influenza A2 virus lung infection, ${ }^{5}$ hydrocarbon-based solvents, ${ }^{6}$ and the nail-patella syndrome. ${ }^{7}$ To our knowledge, no association with neoplasia has been postulated.

In $5-10 \%$ of older patients developing membranous glomerulonephritis, a neoplasm may be the source of the antigen in the glomerular immune complexes; ${ }^{8,9}$ endocrine tumours have not been described in this context. However, pathogenetic mechanisms in membranous glomerulonephritis are quite different from those in Goodpasture's syndrome; in the former, discrete immune complexes are deposited or formed within the glomerular capillary membranes, whereas in the latter, the glomerular basement membrane acts as a fixed antigen to which circulating antibodies attach. Very rarely, both membranous glomerulonephritis and anti-GBM antibody disease have been reported in the same patient, ${ }^{10-12}$ but there is no ultrastructural evidence to support this possibility in our patient.

A basement membrane producing mouse tumour, the EHS sarcoma, may be used as an antigenic substrate for Goodpasture's syndrome. ${ }^{13}$ This tumour contains both Type IV collagen and laminin, neither of which is considered to be the antigenic GBM component, and also one or more glycoproteins which require further investigation. Recently, Pusey and colleagues have described a monoclonal antibody which reacts against a single autoantigenic component distributed throughout basement membranes, ${ }^{14}$ this antibody recognizes the same GBM antigenic component as autoantibodies from patients with Goodpasture's syn- 
drome. The antigen resides within the $\mathrm{NCl}$ domain of basement membrane type IV collagen. To investigate the possibility that an antigen, recognized in the kidney by linear IgG staining of glomerular capillary basement membrane, was produced by or was crossreactive with either of the endocrine tumours described, paraffin-embedded sections were examined by an indirect immunoperoxidase technique for the main immunoglobulin classes. The bronchial carcinoid failed to react with any of the immunoglobulins and unfortunately, insufficient pancreatic tumour tissue remained after sectioning to allow assessment. In view of the known high anti-GBM antibody level at the time of death, this implies that either the pancreatic islet cell tumour was the source of antigen or that neither tumour bore, after routine processing, a crossreactive IgG-type antigenic substrate.

Endocrine cells occur in many parts of the body outside the endocrine system, and occasionally give rise to hyperplasia and neoplasia, both benign and malignant. ${ }^{15}$ They secrete various products, mainly polypeptides but also amines. Bronchial carcinoids, which account for about $5 \%$ of all primary bronchial neoplasms, are occasionally argyrophilic but do not usually secrete active polypeptide hormones. ${ }^{16}$ They are slow-growing, tend to remain localized and rarely metastasize. Pancreatic islet tumours may elaborate various secretory products, both appropriate [insulin, glucagon, somatostatin, pancreatic polypeptide (PP) and vasoactive intestinal polypeptide (VIP)] and inappropriate (ACTH, gastrin and calcitonin). ${ }^{15}$ Macroscopically, they are small and often hard to localize; they are usually argyrophilic on special staining.

Multiple endocrine hyperplasia and neoplasia are well recognized. ${ }^{15}$ They occur in two main categories: MEN (multiple endocrine neoplasia) syndrome Type 1, where a combination of pituitary adenoma, parathyroid hyperplasia, adenoma or carcinoma and pancreatic hyperplasia, adenoma or more rarely, carcinoma is seen, and MEN Type 2, which is subdivided into (a) Sipple's syndrome - a combination of medullary carcinoma of thyroid, phaeochromocytoma of adrenal medulla and parathyroid hyperplasia or neoplasia and (b) where the parathyroid lesions of Type $2 \mathrm{a}$ are replaced by multiple ganglioneuromas and some features of Marfan's syndrome.

The possibility that the endocrine tumours described in this case represent metastases from one site to the other needs to be considered but this is unlikely in view of the difference in histological pattern and the argyrophilia of the pancreatic tumour. None of the more usually described MEN syndromes was present in this case, and the pituitary, thyroid, parathyroid and adrenal glands were normal macroscopically and histologically.

The chemically active secretory products of endocrine tumours are of amine or polypeptide type and not glycoprotein in nature. However, all mammalian cell walls are rich in glycoproteins, and therefore the lack of obvious hormonal secretion by these neoplasms does not preclude the possibility that they served either singly or together as an antigenic source for cross-reactivity with alveolar/glomerular capillary basement membranes. The failure to identify IgG in the bronchial carcinoid tends to suggest that the association between these neoplasms and Goodpasture's syndrome is coincidental rather than causal.

\section{References}

1. Brierley, J., Lawler, W. \& Williams, G. Rapid method for stained renal biopsy specimens embedded in epoxy resin. J Clin Pathol 1987, 41: 1257-1259.

2. Zollinger, H.U. \& Mihatsch, M.J. Renal changes in Goodpasture's syndrome. In: Renal Pathology in Biopsy. Springer Verlag, Berlin, 1978, pp. 337-342.

3. Darmady, E.M. \& MacIver, A.G. Goodpasture's syndrome. In: Renal Pathology. Butterworth and Co., London, 1980, pp. 321-326.

4. Germuth, F.G. \& Rodriguez, E. Immune complex deposits and anti-basement membrane disease. In: Immunopathology of the Renal Glomerulus. Little Brown and Co.., Boston, 1973, p. 183.

5. Wilson, C.B. \& Smith, R.C. Goodpasture's syndrome associated with influenza $\mathrm{A}_{2}$ virus infection. Ann Int Med 1972, 76: $91-94$.

6. Beirne, G.J. \& Brennan, J.T. Glomerulonephritis associated with hydrocarbon solvents - mediated by anti-glomerular basement membrane antibody. Arch Environ Health 1972, 25: 365-369.

7. Curtis, J.J., Bhathena, D., Leach, R.P., Galla, J.H., Lucas, B.A. \& Luke, R.G. Goodpasture's syndrome in a patient with the nail-patella syndrome. Am J Med 1976, 61: 401-406.

8. Stilmant, M.M. Crescentic glomerulonephritis: general comments on EM and IF. In: Rosen, S. (ed.) Pathology of Glomerular Disease. Churchill Livingstone, New York, 1983, pp. 35-61.

9. Kaplan, B.S., Klassen, J. \& Gault, M.H. Glomerular injury in patients with neoplasia. Ann Rev Med 1976, 27: $117-125$.

10. Klassen, J., Elwood, C., Grossberg, A.L. et al. Evolution of membranous nephropathy into anti-gomerular basement membrane glomerulonephritis. N Engl J Med 1974, 290: $1340-1344$.

11. Moorthy, A.V., Zimmerman, S.W., Burkholder, P.M. \& Harrington, A.R. Association of crescentic glomerulonephritis with membranous glomerulopathy: a report of three cases. Clin Nephrol 1976, 6: 319-325. 
12. Lang, C.H., Brown, D.C., Stacey, N. et al. Goodpasture syndrome treated with immunosuppression and plasma exchange. Arch Int Med 1977, 137: 1076-1078.

13. Wick, G., Munro, V., Gebhart, W. \& Timpl, R. Basement membrane-producing tumors as antigenic substrate for the demonstration of anti-basement membrane antibodies. Ann NY Acad Sci 1983, 420: 267-274.

14. Pusey, C.D., Dash, A., Kershaw, M.J., Morgan, A., Rees, A.J. \& Lockwood, C.M. A single autoantigen in Goodpasture's syndrome identified by a monoclonal antibody to human glomerular basement membrane. Lab Invest 1987, 56: 23-31.
15. Dawson, I.M.P. Diffuse endocrine and neuroendocrine cell tumours. In: Anthony, P.P., MacSween, R.N.M. (eds) Recent Advances in Histopathology, Volume 12. Churchill Livingstone, Edinburgh, 1984, pp. 111-128.

16. Spencer, H. Bronchial carcinoid. In: Pathology of the Lung. Pergamon Press, Oxford, 1985, pp. 934-936. 